\title{
Cortical Regions Involved in the Generation of Musical Structures during Improvisation in Pianists
}

\author{
Sara L. Bengtsson ${ }^{1}$, Mihály Csíkszentmihályi ${ }^{2}$, and Fredrik Ullén ${ }^{1}$
}

\begin{abstract}
Studies on simple pseudorandom motor and cognitive tasks have shown that the dorsolateral prefrontal cortex and rostral premotor areas are involved in free response selection. We used functional magnetic resonance imaging to investigate whether these brain regions are also involved in free generation of responses in a more complex creative behavior: musical improvisation. Eleven professional pianists participated in the study. In one condition, Improvise, the pianist improvised on the basis of a visually displayed melody. In the control condition, Reproduce, the participant reproduced his previous improvisation from memory. Participants were able to reproduce their improvisations with a high level of accuracy, and the contrast Improvise versus Reproduce was thus essentially matched in terms
\end{abstract}

\section{INTRODUCTION}

Creative behaviors and creative individuals fascinate scientists and laymen alike, and studies of creativity have a long history in psychology (see, e.g., Simonton, 1999; Sternberg, 1999; Csikszentmihalyi, 1997; Eysenck, 1995). A precise, generally agreed-upon definition of what constitutes a creative behavior has been difficult to arrive at, however, although two characteristics seem central: Creative acts are novel or original and, qualified judges will agree that they constitute valuable contributions to the field (Sternberg, 1999). The novelty aspect is critical, and tests designed to measure creative ability typically require divergent thinking, as opposed to the convergent problem-solving abilities measured by traditional intelligence tests. Convergent problems have a single answer. Divergent tasks have a large number of possible solutions, and the free generation and selection of alternatives among these possibilities are fundamental processes in creative behavior (Campbell, 1960).

Although psychological research has provided valuable information on both creativity as a trait and the characteristics of divergent thinking, little is still known about the brain mechanisms underlying creative behaviors. A few neuroimaging studies have investigated

${ }^{1}$ Karolinska Institutet, Stockholm, Sweden, ${ }^{2}$ Claremont Graduate University of motor output and sensory feedback. However, the Improvise condition required storage in memory of the improvisation. We therefore also included a condition FreeImp, where the pianist improvised but was instructed not to memorize his performance. To locate brain regions involved in musical creation, we investigated the activations in the Improvise-Reproduce contrast that were also present in FreeImp contrasted with a baseline rest condition. Activated brain regions included the right dorsolateral prefrontal cortex, the presupplementary motor area, the rostral portion of the dorsal premotor cortex, and the left posterior part of the superior temporal gyrus. We suggest that these regions are part of a network involved in musical creation, and discuss their possible functional roles.

more complex verbal tasks involving divergent thinking, such as story generation (Howard-Jones, Blakemore, Samuel, Summers, \& Claxton, 2005), sentence completion (Nathaniel-James \& Frith, 2002), generation of unusual verbs in response to nouns (Seger, Desmond, Glover, \& Gabrieli, 2000), and the Brick test of unusual uses of objects (Carlsson, Wendt, \& Risberg, 2000). These studies generally show an activation of cortical association areas, in particular, the prefrontal cortex, during divergent thinking. Interestingly, there appears to be a tendency for the right prefrontal cortex to be particularly involved (Howard-Jones et al., 2005; Carlsson et al., 2000; Seger et al., 2000; Abdullaev \& Posner, 1997). A major difficulty in the investigation of more complex actions, however, is obviously to isolate the neurocognitive components responsible for controlling different aspects of the behavior.

A systematic investigation of neural processes underlying free selection has been performed using simpler model behaviors, as a part of studies of willed action. Willed actions involve "free" choice as well as attention, conscious awareness, and intentionality (Jahanshahi \& Frith, 1998). By studying tasks such as finger or hand movements (Lau, Rogers, Ramnani, \& Passingham, 2004; Frith, 2000; Playford et al., 1992; Deiber et al., 1991) or number generation (Jahanshahi, Dirnberger, Fuller, \& Frith, 2000), and by comparing pseudorandom generation of responses with stereotyped actions, a number of 
cortical regions involved in free selection have been characterized. These include the dorsolateral prefrontal cortex (DLPFC), medial and lateral premotor areas, and the anterior cingulate cortex. This approach has enabled an elegant analysis of the various processes involved in free selection, such as attention to action, working memory, suppression of stereotype responses, and selection per se (Lau et al., 2004; Nathaniel-James \& Frith, 2002; Desmond, Gabrieli, \& Glover, 1998).

However, an interesting question is whether the brain regions involved in free selection in simple willed actions are also utilized in more complex behaviors that could qualify as ecologically valid examples of creativity. In the present study, we investigated this issue using musical improvisation in professional pianists as a model. Improvisation arguably satisfies the demands of a prototypical creative behavior. It involves freely generated choices, but these must be adapted to ongoing performance, and monitored through auditory and somatosensory feedback, as well as to an overall aesthetic goal (Pressing, 1988). It is simple enough, however, to allow an experimental design where the neural processes involved in the free generation of musical structures can be separated from those involved in the sequential organization and programming of the movements (i.e., piano playing), and the processing of movement feedback. To achieve this, we used one condition, Improvise, where the pianist improvised on the basis of a visually displayed melody. In the control condition, Reproduce, the participant reproduced his previous improvisation from memory. The critical contrast, Improvise-Reproduce, was thus matched in terms of motor output and sensory feedback. Because the Improvise condition required storage of the improvisation in memory, we also included a third condition, FreeImp, where the pianist improvised but was instructed not to memorize his performance. To find brain regions involved in music generation, but not in memorization or motor programming, we examined which activations in the Improvise-Reproduce contrast were also present in a conjunction analysis between Improvise-Reproduce and FreeImp-Rest. Activity in the Improvise-Reproduce contrast was regressed on a measure of improvisation complexity in order to localize brain regions with a higher level of activity during the generation of more complex musical structures. Finally, differences in brain activity between FreeImp and Improvise were evaluated by contrasting these two conditions in brain regions that were active in Improvise-Reproduce.

\section{METHODS}

\section{Participants}

Eleven professional Swedish concert pianists took part in the study. All participants were men, healthy, righthanded (Oldfield, 1971), and had a Master's degree in the performing arts (piano) from the Royal Academy of Music in Stockholm. They were between 23 and 41 years old, with a mean age of $32.0 \pm 6.0$ years, and had started playing the piano between 4 and 8 years old (mean $5.7 \pm$ 1.4 years). The experimental procedures were ethically approved by the Karolinska Hospital Ethical Committee (Dn 02-194) and were undertaken with the understanding and written consent of the participants.

\section{Experimental Setup}

Magnetic resonance (MR) imaging recordings were conducted on a 1.5-T scanner (Signa Horizon Echospeed, General Electric Medical Systems). During the functional magnetic resonance imaging (fMRI) scans, the participants played with their right hand on a small piano keyboard, especially designed for usage in an MR environment (LUMItouch, Inc.). The keyboard had one octave of 12 authentic keys (from F to E), and was connected to a PC computer through a fiber-optic cable. During scanning, the participant's performance on the keyboard was recorded on the PC, using the E-Prime software (Psychological Software Tools, Inc.). Auditory feedback from the piano was provided to the participant through headphones. The pianists were lying in a supine position, with the arm supported so that the keyboard could be played by moving the fingers and the wrist without arm movements. A plastic bite bar was used to restrict head movements.

A projector located outside the scanner room was used to project task instructions and musical scores onto a semitransparent screen, positioned approximately $3 \mathrm{~m}$ from the participants' eyes. The participants viewed the screen through a custom-made binocular/mirror system (Lorentzen Instrument $\mathrm{AB}$ ) mounted directly on top of the head coil.

\section{Conditions}

All participants performed three conditions: Improvise, Reproduce, and Rest. These were performed in trials lasting $40 \mathrm{sec}$. During the first $4 \mathrm{sec}$ of a trial, the name of the condition was projected onto the screen. For the Rest condition, the screen after this went blank for the remaining $36 \mathrm{sec}$ of the trial. For the other conditions, a musical score consisting of a simple eight-bar melody was displayed (Figure 1A). For a preparatory period of $8 \mathrm{sec}$, the score was surrounded by a red, rectangular frame. This frame was then removed, signaling to the participant to start playing. These final $28 \mathrm{sec}$ of the trial, when the task was performed on the keyboard, were later used in the data analysis. A total of 12 musical scores of similar complexity were used in the study, 6 in $\mathrm{F}$ major, 6 in $\mathrm{F}$ minor. The main reason to use improvisation based on melodic templates, rather than completely free improvisation, was to make the improvisations 


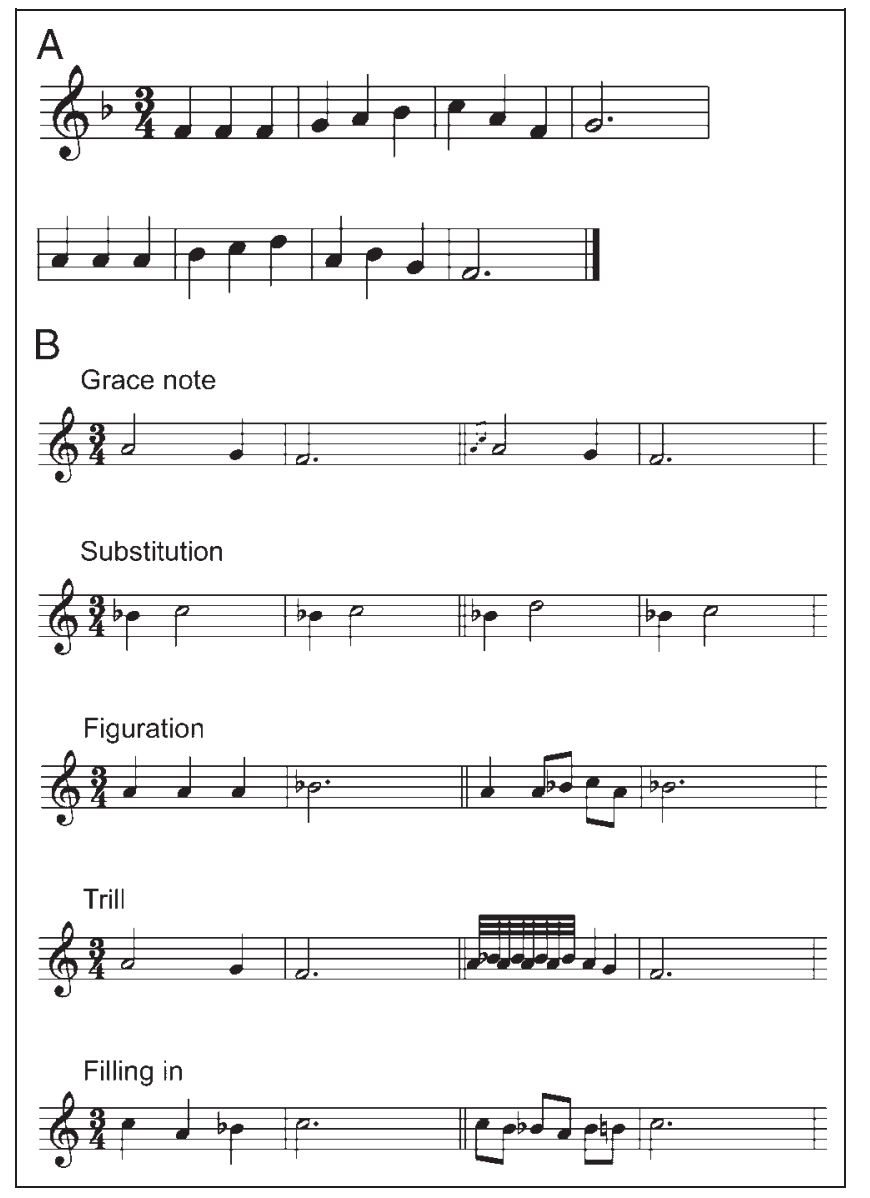

Figure 1. Examples of a musical template and ornamentations used during improvisation. (A) One of the musical templates presented to the participants. (B) The five most common types of modification of templates used in Improvise and FreeImp. Two bars of the original melody are shown to the left of the double bar line, and the improvised modification to the right.

more constrained, and thus, easier to remember. The scores were especially written for the present study, and thus, were unfamiliar to the participants. They were notated using the Finale music notation software (MakeMusic, Inc.).

In Improvise, the displayed melody was used as a basis for an improvisation. The instructions to the participants were to employ any kind of modifications of the presented melodic template they wished, and when the improvisation was finished, to rest without any active movements until the next condition began (for examples of the employed modifications, see Figures $1 \mathrm{~B}$ and 3A-B). They were also instructed to memorize the performance for subsequent reproduction. In Reproduce, the task was to reproduce, as faithfully as possible, the improvisation previously made upon the same melody. In Rest, the participants relaxed, viewing the screen without any movements. Five of the participants performed an additional condition, FreeImp, where the instruction was to improvise on the melody, as in Improvise, but without trying to memorize the performance.

\section{Experimental Procedure}

Before the experiment, the participants were familiarized with the tasks and were given one practice trial of each condition outside the scanner room. When the participant was lying in the MR-scanner in a supine position, the conditions were practiced a second time. The pianists found the tasks enjoyable and interesting. The musical examples used during the practicing sessions were not used again during the experiment.

We started the experiment by collecting a highresolution T1-weighted anatomical image volume of the whole brain. Thereafter, fMRI data were recorded while the participants performed three sessions, each containing three trials of the different tasks. Musical scores were selected randomly from the database of 24 scores. For the same participant, a particular melody was only used once in each of the four conditions. Four such trials, where the same score was used, were always performed consecutively. To minimize task order effects, four different task orders were used in different sessions. A necessary constraint on the task order was obviously that Improvise had to be performed before Reproduce.

\section{MRI Scanning Parameters}

The imaging parameters for the three-dimensional T1weighted image were as follows: field of view, $22 \mathrm{~cm}$; echo time, $6 \mathrm{msec}$; repetition time, $24 \mathrm{msec}$; flip angle, $35^{\circ}$; and voxel size, $0.86 \times 0.86 \times 2 \mathrm{~mm}$. Functional imaging data were recorded as gradient-echo, echo-planar (EPI) T2*-weighted images with blood oxygenation level dependent (BOLD) contrast. Image volumes of the whole brain were built up from 30 continuous axial slices. The following parameter values were used: field of view, $22 \mathrm{~cm}$; echo time, $60 \mathrm{msec}$; repetition time, $4 \mathrm{sec}$; flip angle, $90^{\circ}$; pixel size, $3.4 \times 3.4 \mathrm{~mm}$; slice thickness, $5.0 \mathrm{~mm}$; matrix size, $64 \times 64$. During one session, 122 image volumes were collected continuously. At the beginning of the session, four "dummy" image volumes were scanned, but not saved, to allow for T1 equilibration effects.

\section{Analysis of Behavioral Data}

The onset time and identity of all keys played during scanning were recorded on the PC. The main purpose of the behavioral analysis was to determine how accurately participants reproduced a previous Improvise trial in the Reproduce condition. For this purpose, we performed three types of analysis.

First, the total number of keys played in each trial were calculated. Paired $t$ tests were employed to analyze differences in this parameter for corresponding trials of Improvise and Reproduce (i.e., trials where the same 
musical score was presented). This analysis was performed on all the trials pooled together, as well as separately for the individual participants. Secondly, the same analyses were performed on the total duration of the performance in each trial. For descriptive purposes, number of played keys and duration were calculated also for the FreeImp trials.

Finally, we evaluated the structural similarity of the performances in corresponding trials of Improvise and Reproduce. The Levenshtein edit distance (LED) is a measure of the degree of similarity between two arbitrary sequences (Levenshtein, 1966). It is defined as the minimum number of single element deletions, insertions, or substitutions required to transform one of the sequences into the other. For example, the LED between the two sequences $\mathrm{F}-\mathrm{A}-\mathrm{C}$ and $\mathrm{F}-\mathrm{G}$ is 2 ( $\mathrm{F}-\mathrm{A}-$ $\mathrm{C} \rightarrow \mathrm{F}-\mathrm{G}-\mathrm{C} \rightarrow \mathrm{F}-\mathrm{G})$. It can easily be seen that the LED is at least as large as the difference in length between the two sequences, and 0 only in the case of identical sequences. The LED between different melodic structures, that is, the sequences of played keys, was calculated using a standard algorithm (Knuth, 1981) implemented in Matlab 6 (The MathWorks, Inc.). The LED between corresponding Improvise-Reproduce trials was investigated as a measure of the accuracy of the improvisations. The LED between each improvisation and its original template melody was used as a measure of the complexity of the improvisation. All statistical analyses were performed in Statistica (StatSoft, Inc.).

\section{Processing and Statistical Analysis of the fMRI Data}

The fMRI data were analyzed using the SPM99 software package (Wellcome Department of Imaging Neuroscience, London, UK). The scanned brain volumes were realigned to correct for head movements. Subsequently, they were coregistered to each individual's T1-weighted image (Ashburner \& Friston, 1997) and normalized to the standard space (Talairach \& Tournoux, 1988). Proportional scaling was applied to eliminate the effects of global changes in the signal. The time series were smoothed spatially with an isotropic Gaussian filter of $10 \mathrm{~mm}$ full width at half-maximum, and temporally with a Gaussian kernel of width 4 sec.

The fMRI data were modeled with the general linear model, where we defined four conditions of interest corresponding to the periods in each epoch when the participants performed the task (the last $28 \mathrm{sec}$ of the 40-sec epochs). We modeled the first $12 \mathrm{sec}$ of each epoch (i.e., the presentation of the task instruction and the preparatory period) as conditions of no interests. The significance of the effects was assessed using onetailed $t$ statistics for every voxel from the brain image, to create statistical parametric maps, which were transformed into $Z$ statistics. Analyses were performed for contrast subtractions of interest within participants, followed by a between-participants random-effects analysis based on summary-statistics of the subtraction images created for each participant. In this way, the interparticipant variance was accounted for, and inferences can be extended to the population from which the participants are drawn.

To localize brain regions involved in real-time improvisation and recall of a previous improvisation from memory, respectively, the contrasts Improvise-Reproduce and Reproduce-Improvise were investigated. To exclude the possibility that differences in these conditions reflected a deactivation, the contrasts Improvise-Rest and Reproduce-Rest, respectively, were used as inclusive masks. For both masks, an uncorrected $p$ value of .05 was used. To investigate whether the brain activity seen in Improvise-Reproduce could reflect reproduction errors, a regression analysis was performed across participants, to test if the parameter estimates for the ImproviseReproduce contrast correlated with the mean LED between improvisations and reproductions for all trials performed by each participant. In a second betweensubject regression analysis, Improvise-Reproduce activity was regressed on the mean LED between the original melodic template and improvisation, in order to localize brain activity related to the generation of more complex improvisations.

Brain activity in the Improvise-Reproduce contrast could reflect storage in memory, as participants were required to reproduce the Improvise trials during Reproduce. We therefore investigated which of the activations in Improvise-Reproduce were also seen in the contrast FreeImp-Rest, using a conjunction analysis between these two contrasts. We utilized a "minimum statistic compared to the conjunction null" analysis, as described in Nichols, Brett, Andersson, Wager, and Poline (2005), which can be interpreted as a logical ANDoperation between the two contrasts. Because only five participants performed the FreeImp condition, the conjunction was analyzed using a fixed-effects model to increase statistical sensitivity. Finally, to investigate differences in activity between FreeImp and Improvise in regions that were involved in improvisation, the contrasts FreeImp-Improvise and Improvise-FreeImp were examined. Small volume corrections for multiple comparisons were employed, with spherical regions of interest (radius $10 \mathrm{~mm}$ ) centered around the peak coordinates of the activations in Improvise-Reproduce.

We report activations that were significant at $p<.05$ according to a False Discovery Rate (FDR) analysis (Genovese, Lazar, \& Nichols, 2002). In this analysis, the results are corrected for multiple comparisons and a threshold is set to control the rate of false positives. The threshold of $p<.05$ thus means that, on average, less than $5 \%$ of the suprathreshold voxels are not truly active. For the contrast Improvise-Reproduce, no activity was found at this threshold in the presupplementary motor area (pre-SMA). However, a recent study by Lau 
et al. (2004) found the pre-SMA to be the one region where a parametrical relation between brain activity and performance in a free selection task was found. We therefore used a small volume correction within a spherical region of interest (radius $10 \mathrm{~mm}$ ) in the preSMA, using the coordinate of the peak of activity $(x, y$, $z=8,16,64)$ in Lau et al. as the center of the sphere. Anatomical localizations of the activated regions were determined from an average image of normalized and intensity standardized T1-weighted images from all 11 participants. We used the anatomical terminology of Duvernoy (2000). To localize cerebellar activations, we used the atlas of Schmahmann, Doyon, Toga, Petrides, and Evans (2000).

\section{RESULTS}

\section{Descriptive Characteristics of the Improvisations in Improvise and FreeImp}

The identity and onset time of all piano keys played during scanning were recorded. Each condition was performed nine times, each time with a different melodic template, by each of the 11 participants, giving a total of 99 trials each of Improvise and Reproduce. During three of these trials (each in a different participant), a temporary mechanical error in the keyboard prevented proper recording of the behavioral data. Ninety-six trials of Improvise and Reproduce are thus included in the analysis below. FreeImp was performed by five subjects. Here, two trials in two different subjects had to be discarded for technical reasons, giving a total of 43 trials included in the analysis. The displayed melodic templates had, on average, $17.0 \pm 2.6$ (mean $\pm S D$ ) notes.

For Improvise trials, the total number of played keys was $29.1 \pm 9.4$, and the mean duration of the improvisations was $17.8 \pm 3.2 \mathrm{sec}$. The mean LED between the melodic template and improvisation was $20.7 \pm 10.6$. The improvisations in FreeImp were slightly more elaborate than in Improvise. They contained a larger number of notes (mean number of played keys $36.4 \pm 9.2$ ) than the corresponding performances in Improvise [paired $t$ test: $t(42)=6.02 ; p=.000]$. The total duration of the FreeImp improvisations was also longer (18.4 \pm $3.6 \mathrm{sec}$ ) than in Improvise, although this trend did not reach significance [paired $t$ test: $t(42)=1.84 ; p=.07$ ] . The LED between FreeImp improvisations and their templates was higher $(27.8 \pm 9.6)$ than for Improvise [paired $t$ test: $t(42)=4.12 ; p=.000$ ].

During both Improvise and FreeImp, participants always played a modified version of the entire original melodic template, as written, that is, in no cases were only a part of the template or transformed (e.g., retrograde, mirrored) versions of template utilized as a basis for the improvisation. A qualitative analysis of all improvisations revealed that all modifications could be classified into 11 categories (Table 1): (i) Insertion of a
Table 1. Modifications of the Melodic Templates Used in Improvise and FreeImp

\begin{tabular}{lrrrrr}
\hline & \multicolumn{2}{c}{ Improvise } & & \multicolumn{2}{c}{ FreeImp } \\
\cline { 2 - 3 } \cline { 6 - 7 } Modification & $n$ & \% of Total & & $n$ & \% of Total \\
\hline Grace note & 247 & 40.1 & & 157 & 46.4 \\
Substitution & 112 & 18.2 & & 76 & 22.5 \\
Figuration & 74 & 12.0 & & 31 & 9.2 \\
Trill & 73 & 11.9 & & 35 & 10.4 \\
Filling in & 38 & 6.2 & & 2.7 \\
Repetition & 23 & 3.7 & & 13 & 3.8 \\
Elimination & 23 & 3.7 & & 2.7 \\
Two part & 12 & 1.9 & & 3 & 0.9 \\
Rhythmization & 10 & 1.6 & & 3 & 0.9 \\
Tremolo & 3 & 0.5 & & 1 & 0.3 \\
Minor/major shift & 1 & 0.2 & & 1 & 0.3 \\
Total & $\mathbf{6 1 6}$ & 100 & 338 & 100 \\
\hline
\end{tabular}

For each condition, the total number of modifications of a particular type $(n)$, as well its relative frequency (\% of total), is shown.

fast group of one or more grace notes before a template note; (ii) substitution of a template note for another note; (iii) figuration, that is, expansion of the original template into melodic figures; (iv) insertion of a trill on a template note; (v) filling in, that is, insertion of chromatic or diatonic scales between template notes; (vi) repetitions of template notes; (vii) elimination of template notes; (viii) insertion of figures giving a broken two-part polyphony; (ix) rhythmization of the template; (x) insertion of a tremolo, that is, a trill-like figure between two notes with a larger interval than a second; and finally, (xi) switching of tonality from major to minor. Examples of the five most common types of modification (i-v), which together constitute more than $88 \%$ of the modifications in both Improvise and FreeImp, are shown in Figure 1B.

The larger number of notes in the FreeImp improvisations was due to a larger mean number of modifications per improvisation $[t$ test: $t(137)=3.40 ; p=.001]$ in FreeImp $(7.9 \pm 2.5)$ than in Improvise $(6.4 \pm 2.2)$. No differences in the relative frequencies of the 11 different modifications (Table 1) were found between the two conditions [paired $t$ test: $t(10)=0.000 ; p=1.0$ ], nor was the mean number of notes per modification different $[t$ test: $t(134)=0.30 ; p=.77]$ in Improvise $(5.3 \pm$ 3.0) and FreeImp $(5.5 \pm 4.2)$.

\section{Accuracy of the Reproductions}

Pooling data from all participants, no significant difference [paired $t$ test: $t(95)=1.58 ; p=.12$ ] was found 
between the total number of keys played in Improvise and Reproduce trials (29.6 \pm 9.8). Similarly, no difference [paired $t$ test: $t(95)=1.51 ; p=.13$ ] was found in the total mean duration of Improvise and Reproduce $(17.6 \pm 3.4 \mathrm{sec})$ trials. Nor could any significant differences in these variables be found within single participants. The mean number of played keys per trial in Improvise and Reproduce is shown for each participant in Figure 2A. When analyzing all participants individually with repeated paired $t$ tests, no significant differences were found (all Bonferroni-corrected $p$ values $>.20$ ). Individual data for trial durations are shown in Figure 2B. No significant differences were found in the durations using paired $t$ tests within each participant (corrected $p$ values $>.52$ ).

In terms of overall motor output-number of key strokes and duration of the performances-participants were thus highly consistent in Improvise and Reproduce. To further investigate how well each improvisation was reproduced structurally, we calculated the LED between the key sequences played in corresponding trials of Improvise and Reproduce (see Methods). The mean LED for all trial pairs was $7.5 \pm 4.9$. Many of these single key edits were due to minor, and musically irrelevant, differences for instance, in the number of notes included in a trill or other ornament. An example

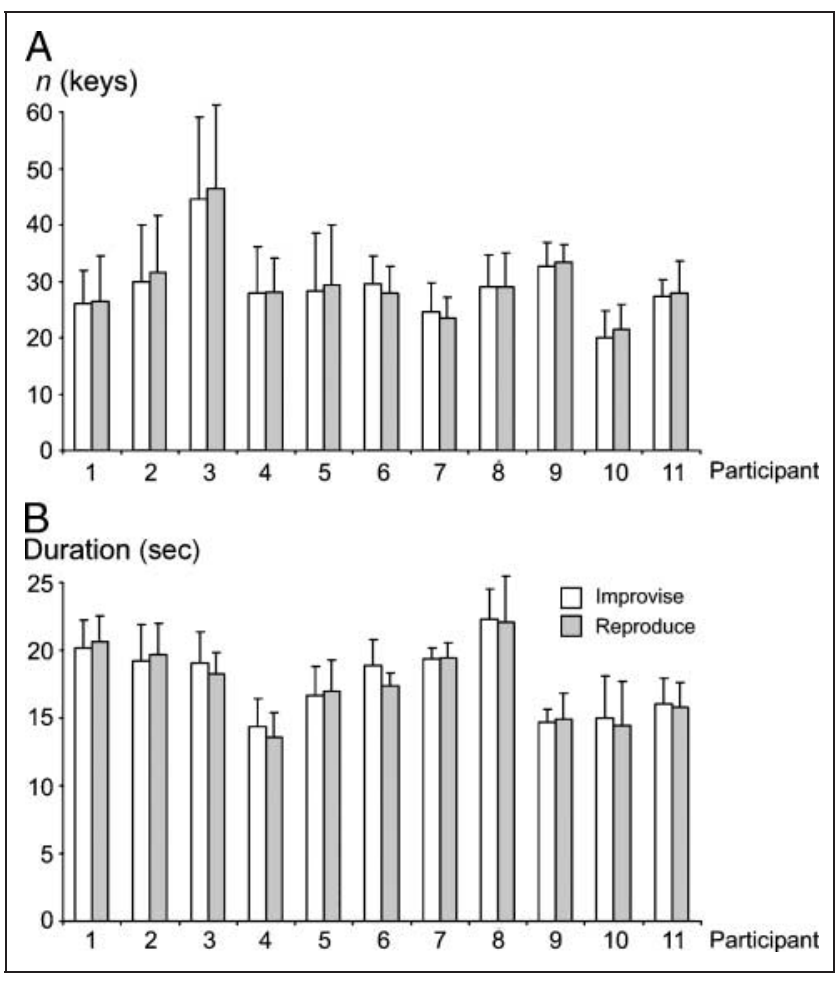

Figure 2. Behavioral data recorded during the scanning. (A) The mean number of played keys per trial in Improvise and Reproduce for each individual participant. (B) The mean duration of Improvise and Reproduce trials for each participant. In both A and B, error bars indicate standard deviations. of an Improvise-Reproduce trial pair where the reproduction was of average accuracy (LED 8) is illustrated in Figure 3. The diagrams (Figure 3A, C) show the onset time of each key stroke. The same trials are illustrated in musical notation in panels $\mathrm{B}$ and $\mathrm{D}$. The two trials are practically identical in overall conception, and highly similar also in the individual details. In this case, four single edits were due to that fact that one single ornament (encircled) was forgotten in the Reproduce trial. The remaining single key edits are due to minor differences in the execution of the details of other ornaments. In summary, the participants were able to reproduce their performances in Improvise with remarkable accuracy in the Reproduce condition. To further investigate whether differences in reproduction accuracy influenced the observed brain activity, a participant level regression analysis was performed between mean LED and activity in the Improvise-Reproduce contrast (see below).

\section{fMRI Data}

Brain areas with significantly higher BOLD response during Improvise than during Reproduce are summarized in Table 2 and illustrated in Figure 4. The histograms in Figure 4 show, for those subjects who performed all four conditions, the mean percent signal change in BOLD signal for each condition in peak voxels of the active regions, with the Rest condition as zero. In the frontal lobe, activations were found in the right DLPFC and pre-SMA, and bilaterally in the rostral portion of the dorsal premotor cortex (PMD). Temporal lobe activations were found in the left posterior superior temporal gyrus (STG), close to the temporo-parietal junction, and in the fusiform gyrus. Bilateral occipital activity was found in the middle occipital gyrus. For each peak of activity found in this contrast, we have indicated (Table 2, rightmost column) whether the same region was also activated in a conjunction between Improvise-Reproduce and FreeImp-Rest. This was the case for all activations, except for the peak in the left STG and one of the peaks in the left PMD. However, peaks were found in the close vicinity of these regions (Table 2 , footnotes). The durations of the improvisations were variable, and shorter than the 28-sec epoch length (mean duration $17.8 \mathrm{sec}$; see above). To verify that the observed activations were not confounded with brain activity occurring after the improvisations, we therefore also performed a separate analysis where only the first $16 \mathrm{sec}$ of the improvisations were included. All activations in Table 2 were found also in this analysis.

To test whether the activations in Improvise-Reproduce correlated with the accuracy of the reproductions, we regressed single-participant activations in this contrast on the mean LED between improvisations and reproductions in all trials performed by each participant. No significant positive or negative correlations were found. Neither were significant correlations found in the Reproduce- 


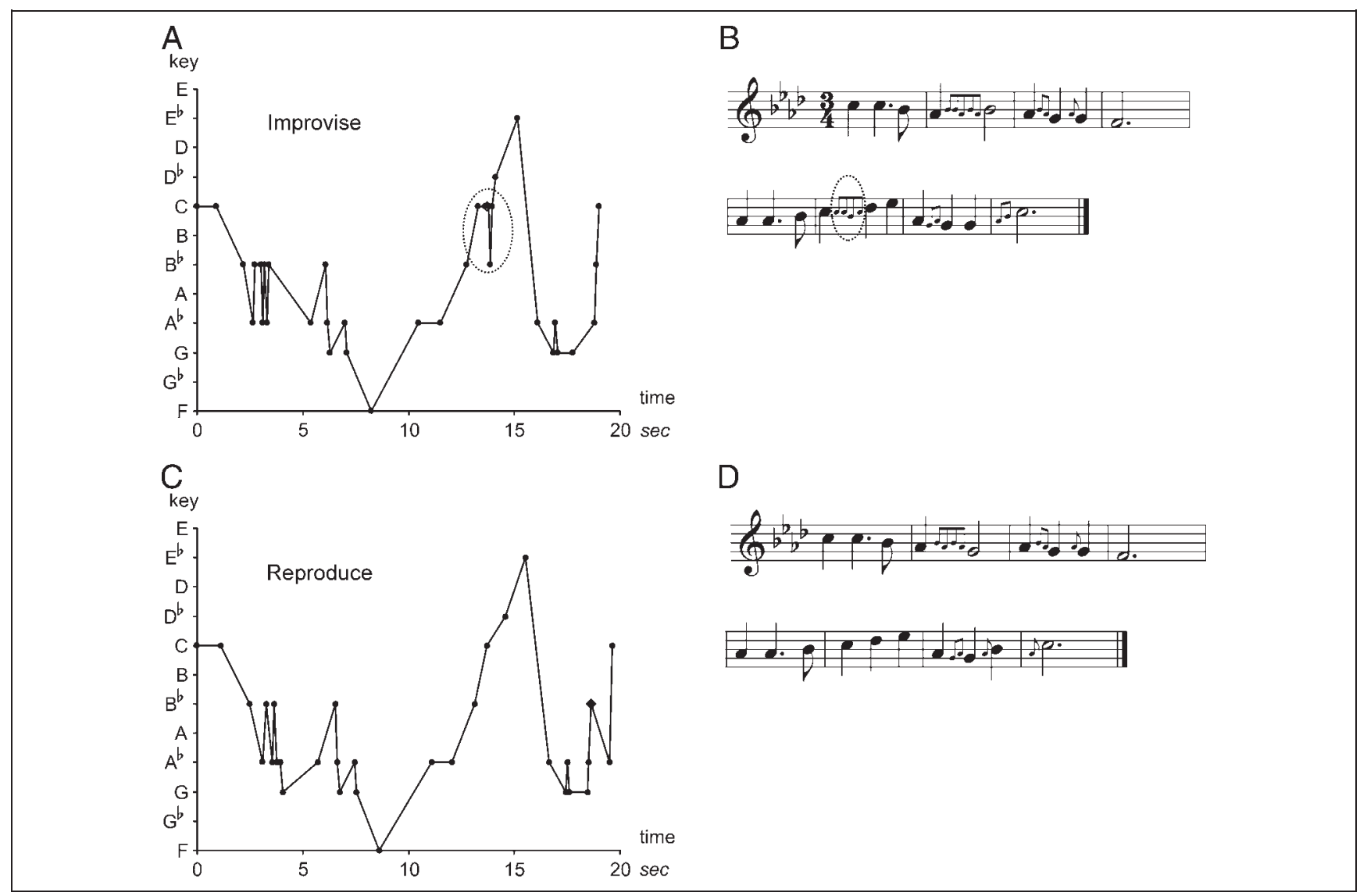

Figure 3. An example of a typical Improvise-Reproduce trial pair. The diagrams (A, C) show the onset time of each key stroke of the performance. The same trials are illustrated in musical notation in panels B and D. One ornament, which was not properly reproduced, is encircled in $\mathrm{A}$ and $\mathrm{B}$.

Improvise contrast at the current threshold (FDR $<0.05)$, or when using a more liberal threshold of FDR $<0.2$. To localize brain activity related to the complexity of the improvisations, brain activity in Improvise-Reproduce was regressed onto the mean LED between improvisations and the original melody template for each participant. A significant positive relation was found in the pre-SMA (Figure 5). The diagram shows the correlation $(r=.71 ; p=.01$; Pearson Product-Moment Correlation) between BOLD activity in the peak coordinate of the cluster $(x, y, z=12,15,54)$ and improvisation complexity. As can be seen, one of the participants produced much more complex improvisations than the rest of the group. When removing this outlier, a positive trend remained $(r=.28)$, but did not reach significance $(p=.43)$.

No activations were significant at $p<.05$ (FDR) in the contrasts FreeImp-Improvise and Improvise-FreeImp. In FreeImp-Improvise, nonsignificant trends were found in the DLPFC ( $p=.27 ; t=2.24 ; x, y, z=30,45,21)$ and the middle occipital gyrus $(p=.12 ; t=2.41 ; x, y$, $z=-42,-81,2)$. In Improvise-FreeImp, nonsignificant trends were seen in the PMD $(p=.15 ; t=2.48 ; x, y, z=$ $24,9,60)$ and the middle occipital gyrus $(p=.07 ; t=$
$2.65 ; x, y, z=-33,-78,27)$. In no other regions were voxels found above a threshold of $p=.05$, uncorrected.

\section{DISCUSSION}

We compared brain activity during on-line improvisation (Improvise) and the reproduction of a previously created improvisation from memory (Reproduce). Three important questions have to be considered when interpreting the Improvise-Reproduce contrast.

First, the pianists did not reproduce their improvisations with perfect accuracy. Does the brain activity in Improvise-Reproduce reflect these minor differences in motor output? Several observations speak against this. The number of played keys did not differ between the two tasks. In fact, a nonsignificant trend was found for a larger total motor output in Reproduce than in Improvise. One could therefore expect brain activity related to differences in motor output to appear in the Reproduce-Improvise contrast, but no significant activations were seen in that comparison. Furthermore, the duration (i.e., time on task) of improvisations and corresponding reproductions did not differ. Finally, corresponding trials were highly similar in terms of 
Table 2. Brain Regions with Significantly Increased BOLD Contrast Signal in Improvise-Reproduce

\begin{tabular}{|c|c|c|c|c|c|c|}
\hline Brain Region & Side & $x$ & $y$ & $z$ & $t$ Value & Conj $^{\mathrm{a}}$ \\
\hline \multicolumn{7}{|l|}{ Frontal lobe } \\
\hline $\begin{array}{l}\text { Middle frontal g. } \\
\text { (DLPFC) }\end{array}$ & $\mathrm{R}$ & 33 & 39 & 27 & 5.24 & + \\
\hline \multirow{4}{*}{$\begin{array}{l}\text { Superior frontal } \\
\text { sulcus (PMD) }\end{array}$} & \multirow[t]{3}{*}{$\mathrm{L}$} & -24 & 12 & 48 & 4.41 & $-{ }^{\mathrm{b}}$ \\
\hline & & -27 & 9 & 60 & 8.73 & + \\
\hline & & -33 & -3 & 60 & 5.06 & + \\
\hline & $\mathrm{R}$ & 27 & 12 & 48 & 6.60 & + \\
\hline $\begin{array}{l}\text { Superior frontal g. } \\
\text { (pre-SMA) }\end{array}$ & $\mathrm{R}$ & 9 & 12 & 54 & $2.88^{c}$ & + \\
\hline \multirow{2}{*}{$\begin{array}{l}\text { Middle frontal g. } \\
\quad(\mathrm{PMD})\end{array}$} & \multirow[t]{2}{*}{$\mathrm{L}$} & -33 & 3 & 42 & 6.14 & + \\
\hline & & -36 & -3 & 45 & 6.58 & + \\
\hline
\end{tabular}

Temporal lobe

Posterior STG $\begin{array}{lllll}\mathrm{L} & -60 & -39 & 15 & 7.42\end{array}$

Fusiform g.

$\begin{array}{llll}45 & -51 & -12 & 4.70\end{array}$

$-{ }^{\mathrm{d}}$

$+$

Occipital lobe

$\begin{array}{lrrrrrr}\text { Middle occipital g. } & \text { L } & -36 & -78 & 18 & 4.20 & + \\ & & -27 & -87 & -3 & 4.21 & + \\ & \text { R } & 39 & -78 & 6 & 4.57 & + \\ & & -81 & 0 & 4.77 & +\end{array}$

${ }^{\mathrm{a}} \mathrm{A}(+)$ sign in this column indicates that activity $(\mathrm{FDR}<0.05)$ in this region was also found in a conjunction between (Improvise-Reproduce) and (FreeImp-Rest)

${ }^{\mathrm{b}}$ The nearest active voxel in the conjunction was found in the L PMD at $-33,6,45(x, y, z)$.

${ }^{\mathrm{c}}$ Significant at FDR $<0.05$ after a small volume correction based on an a priori hypothesis, but not in a whole-brain analysis.

${ }^{\mathrm{d}}$ The nearest active voxel in the conjunction was found in the inferior parietal cortex immediately above the temporo-parietal junction, at $-51,-39,30(x, y, z)$.

sequential structure, and no correlations were found between an index of structural similarity (LED) and brain activity at the single-participant level. Had the activations reflected motor output differences, one would have expected a higher level of activity in participants with a higher mean LED value, as this implies larger discrepancies between the improvisations and the reproductions. For the same reasons, we consider Improvise and Reproduce to be essentially equivalent in terms of sensory input (i.e., auditory and somatosensory feedback).

Secondly, the improvisations were of variable duration. Could activations in the Improvise-Reproduce contrast be confounded with non-task-related brain activity occurring after the performance? Two facts speak against this. First, the same set of activations were seen in the Improvise-Reproduce contrast when analyzing only the first $16 \mathrm{sec}$ of the improvisations. Secondly, participants were instructed to rest passively after finishing the performance.

Thirdly, because the improvisations had to be reproduced, the Improvise condition required both improvisation and storage of the performance in working and long-term memory. To what extent does the neural activity in Improvise-Reproduce reflect the latter processes? To evaluate this question, we investigated which of the activated brain areas were also seen in the contrast FreeImp-Rest. For the FreeImp condition, the participants had been instructed to improvise without memorizing their performance. The fact that the same types of modifications of the template were used with the same relative frequencies in FreeImp and Improvise suggests that no major differences in improvisatory strategies were used in these two conditions. All major regions active in Improvise-Reproduce- the DLPFC, the rostral PMD, the left temporo-parietal region, and the middle occipital gyri-were also active in the conjunction analysis. In summary, we therefore argue that the activity in these brain regions reflects neural processes involved in the generation of new musical material during improvisation.

\section{Dorsolateral Prefrontal Cortex}

A key finding in the present study is that the DLPFC is involved in the generation of musical structures during improvisation. This is of interest because it demonstrates that the DLPFC is involved in the creative aspects of a complex and ecologically relevant behavior, where the free selection of responses is adapted to an overall goal of producing an aesthetically satisfactory end-result.

The finding is in line with the many earlier studies that have used simpler model behaviors to investigate the involvement of the DLPFC in free response selection. The DLPFC is consistently more active during motor tasks when movement parameters such as effector (Frith, Friston, Liddle, \& Frackowiak, 1991), movement direction or target (Rowe, Stephan, Friston, Frackowiak, \& Passingham, 2005; Playford et al., 1992; Deiber et al., 1991), and movement timing (Jahanshahi, Jenkins, Brown, Marsden, Brooks, et al., 1995; Jahanshahi, Jenkins, Brown, Marsden, Passingham, et al., 1995) are freely chosen, as opposed to repetitive or externally determined by a stimulus. Similarly, DLPFC activity is related to free selection in cognitive tasks. This has been shown, for instance, for word generation (Frith et al., 1991; Petersen, Fox, Posner, Nintus, \& Raichle, 1988), number generation (Jahanshahi et al., 2000), word-stem completion (Desmond et al., 1998), and sentence completion (Nathaniel-James \& Frith, 2002). It can also be noted that there was a trend for higher DLPFC activity during FreeImp than during Improvise, which could be related to the slightly higher level of complexity (larger number of modifications) of the improvisations in FreeImp. 
Figure 4. Brain regions active in the Improvise-Reproduce contrast. Activity maps of brain regions with significantly increased BOLD contrast signal are shown for the right dorsolateral prefrontal cortex (DLPFC; slice $y=39$ ), the left superior temporal gyrus (STG; slice $x=-60$ ), and the bilateral dorsal premotor cortices (PMD; slices $y=6$ and $z=45$ ). The color scale shows $t$ values. $\mathrm{R}$ and $\mathrm{L}$ denote the left and right sides, respectively. The histograms show mean percent signal change in BOLD signal for each condition in peak voxels of the active regions, with the Rest condition as zero. Error bars show standard error of the mean (SEM). Names of conditions are abbreviated as follows: $\mathrm{B}=$ Rest (baseline); I = Improvise; $\mathrm{R}=$ Reproduce; $\mathrm{F}=$ and FreeImp.

What are the specific functional roles of the DLPFC in this type of tasks? Part of the activity may relate to attention to the selection of action, rather than free choice per se (Lau et al., 2004; Jueptner et al., 1997). Lau et al. (2004) found that the DLPFC was activated during selection between several possible responses, whether these were externally specified or free. However, other studies have reported a positive relation between the number of available alternatives in a free choice situation and the level of DLPFC activity (Nathaniel-James \& Frith, 2002; Desmond et al., 1998), suggesting that the DLPFC is also involved in the selection process.

It should be noted that "free" in free-selection tasks typically means pseudorandom. The apparent simplicity of such a task is obviously deceptive (Jahanshahi et al., 1998). To emulate randomness, participants must rely on some strategy to produce an irregular output. One task for the DLPFC is thus presumably to maintain earlier responses in working memory. In this way, different response alternatives can be compared to previous output to avoid regularities. The DLPFC is strongly implicated in working memory for action-relevant information (for a review, see Fuster, 2001), and is important for the continuous comparison of consecutive stimuli (Petrides, 1995). Transcranial magnetic stimulation experiments show that disruption of DLPFC activity during pseudorandom generation of numbers (Jahanshahi et al., 1998) or letters (Jahanshahi \& Dirnberger, 1999) tends
Figure 5. The pre-SMA and improvisation complexity. In the pre-SMA, a positive relation was found between brain activity in the ImproviseReproduce contrast, and the degree of complexity of the improvisations, operationalized as the LED between improvisation and template. Adjusted fMRI data from the peak voxel of the cluster (the red cross in the activity map; $x, y, z=12,15$, 54) are plotted against mean improvisation complexity for each participant in the graph. Each dot in the plot represents an individual participant.

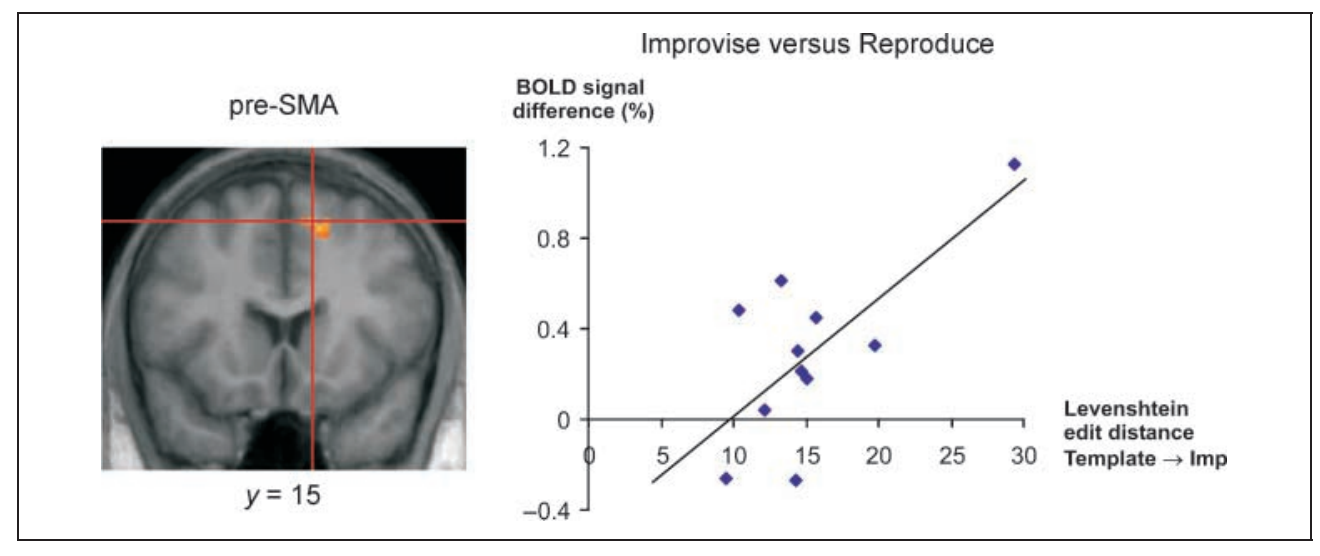


to make the responses more stereotyped. One role of the DLPFC in free selection may thus be to inhibit unwanted, habitual responses.

All these subfunctions of the DLPFC-attention to action, monitoring in working memory, response selection, and suppression of stereotype responses-may be of importance during improvisation. In addition, it appears likely that improvisation, perhaps to a larger degree than attempts at random behavior, relies on the higher, integrative mechanisms of the DLPFC. During Improvise, a whole set of freely selected modifications of the original melody must be temporally organized according to a musically meaningful overall plan. A central role for the DLPFC in planning and performance of novel or complex behavioral sequences, including language and thought, is demonstrated by a vast body of neurological and experimental data (for reviews, see Fuster, 2001; Cummings, 1993; Baddeley, 1986; Luria, 1966). Our data are in line with that one central function of the DLPFC during improvisation is supervisory: to maintain and execute an overall plan for the improvisation through top-down influences on the activity, for instance, in subordinate premotor areas. One aspect of this may be "sculpting of the response space" (Frith, 2000), that is, the selection of a set of responses suitable for a particular improvisation. These influences can be mediated by the extensive connections from the DLPFC to the motor system, including the rostral premotor areas (Fuster, 2001; Picard \& Strick, 2001). Notably, the DLPFC activation was in the right hemisphere. This is consistent with a number of earlier studies which also found predominantly right-sided activity during divergent tasks (Howard-Jones et al., 2005; Carlsson et al., 2000; Seger et al., 2000; Abdullaev \& Posner, 1997).

\section{Rostral Premotor Cortices}

Activity in Improvise-Reproduce was also found in rostral premotor areas, mesially in the pre-SMA as well as laterally in the PMD. Our findings fit the general view that the rostral portion of the premotor cortex is involved in cognitive aspects of action (Picard \& Strick, 2001). Both the pre-SMA (Johansen-Berg et al., 2004; Lu, Preston, \& Strick, 1994; Bates \& Goldman-Rakic, 1993) and the rostral PMD (Lu et al., 1994; Barbas \& Pandya, 1987) are, unlike caudal premotor areas, interconnected with the DLPFC.

The pre-SMA has been implicated in free choice, in particular, when the timing of a response is selected (Jahanshahi, Jenkins, Brown, Marsden, Passingham, et al., 1995). Interestingly, Lau et al. (2004) recently found that not only was the pre-SMA active in free selection but the level of pre-SMA activity also correlated with response time between participants. The finding of a positive relation between pre-SMA activity and the complexity of the improvisations is in line with a role of the pre-SMA in the selection process. This correlation did not remain significant when removing one outlier participant that produced highly complex improvisations. However, because a positive trend was still seen, it seems likely that this reflects a restriction of range in terms of improvisational complexity among the other participants. Our data thus indicate that the pre-SMA is involved in response selection also in more complex divergent tasks such as musical improvisation. The involvement of the pre-SMA in temporal selection is of interest in relation to the consistent finding that this region is active during rhythmic sequence performance (Bengtsson, Ehrsson, Forssberg, \& Ullén, 2004, 2005; Lewis, Wing, Pope, Praamstra, \& Miall, 2004; Schubotz \& von Cramon, 2001; Lutz, Specht, Shah, \& Jäncke, 2000; Larsson, Gulyás, \& Roland, 1996) as well as during perceptual timing tasks (Macar et al., 2002). One could therefore suggest that the pre-SMA activity during improvisation may be particularly related to decisions about timing and rhythmic patterning. In addition, it seems plausible that demands on temporal processing are higher during improvisation, when generated ornaments have to be fitted into a given metric structure, than during reproduction.

The PMD receives a large input from the superior parietal lobule, and plays important roles for visuomotor control, sequencing, and spatially targeted movements (Andersen, Snyder, Bradley, \& Xing, 1997; Wise, Boussaoud, Johnson, \& Caminiti, 1997). As for the medial wall motor areas, more rostral activations are associated with higher-order, non-movement-related processing (Picard \& Strick, 2001). Two findings on the rostral PMD can be mentioned, in particular, in relation to our results. First, the rostral PMD is implied in response selection in visual choice reaction time tasks (Grafton, Fagg, \& Arbib, 1998). Secondly, neurons in this region have been shown to be involved in transforming a series of positional cues kept in working memory, into a sequential motor program of targeted movements (Ohbayashi, Ohki, \& Miyashita, 2003). The rostral PMD could be involved in similar operationsresponse selection based on visual cues, that is, musical notation, and transformation of information held in working memory by the DLPFC and the pre-SMA into movement sequences-during improvisation. The trend for higher PMD activity during Improvise than during FreeImp could reflect a higher load on working memory in Improvise, where the participants had to memorize their performance.

Earlier work on movement sequence production has indicated that medial premotor areas are more important for timing, whereas lateral premotor cortex activity is more related to sequencing of the movements in the correct order (Bengtsson et al., 2004; Schubotz \& von Cramon, 2001). An interesting possibility is that a similar division of labor holds also during improvisation so that the rostral PMD and the pre-SMA are more involved in the shaping of melodic and rhythmic structures, respectively. 


\section{Temporal and Occipital Areas}

We found activity specifically related to Improvise in a portion of the posterior STG, close to the temporo-parietal junction. This region, area Spt (Hickok, Buchsbaum, Humphries, \& Muftuler, 2003), has consistently been found active in studies that require auditory-motor integration, such as rhythmic sequence performance (Bengtsson et al., 2004, 2005; Lewis et al., 2004; Jäncke, Loose, Lutz, Specht, \& Shah, 2000; Lutz et al., 2000) and vocal rehearsal of words or music (Hickok et al., 2003). It can affect the motor system through connections with inferior frontal regions, via the arcuate fasciculus (Hickok \& Poeppel, 2000). We suggest that the area Spt activation in the present study may reflect a larger load on auditory-motor feedback loops during Improvise, for example, to adapt the improvisation to ongoing performance. In addition, the posterior superior temporal cortex is involved in auditory working memory of melodic structures (Gaab, Gaser, Zaehle, Jancke, \& Schlaug, 2003; Patterson, Uppenkamp, Johnsrude, \& Griffiths, 2002; Griffiths, Buchel, Frackowiak, \& Patterson, 1998), and may thus be of importance for auditory monitoring of the ongoing performance. Finally, the different modifications of the original template employed in the improvisations are obviously part of a common "vocabulary" of ornaments used in Western art music (see Palmer, 2001). One possibility is that the area Spt is involved in retrieval of such musical structures from long-term memory, in analogy with its role in lexical retrieval in linguistic tasks (Hickok \& Poeppel, 2000).

A number of small clusters of activity were found in higher-order visual areas in the fusiform and middle occipital gyri. These activations may be due to a more intense visual processing of the musical score when this was used as a basis for improvisations, rather than as a template to recall a previous performance. Notably, two of these regions (right fusiform and left middle occipital gyri) were found to be involved in music reading in another study on the same group of participants (Bengtsson \& Ullén, 2006).

\section{Conclusion}

We have provided evidence that a set of frontal and temporal association areas are specifically involved in the free creation of musical structures during improvisation. We suggest that the DLPFC interacts with the rostral PMD and the pre-SMA in processes of free selection, selective attention, as well as the sequential and temporal organization of the behavior, and with area Spt in the superior temporal cortex for auditory working memory, retrieval of musical standard ornaments from long-term memory, and auditory-motor integration. For the pre-SMA, increased activity related to the generation of more complex improvisations could be demonstrated.
We believe this study demonstrates that musical improvisation may be a useful behavior for studies of the neurocognitive processes underlying an ecologically relevant creative behavior. An important next step will be to analyze the neural underpinnings of the cognitive components of improvisation, such as production of melodic and rhythmic structures, and the interaction between systems for planning, motor attention, response generation, and selection. The brain regions shown to be involved in musical improvisation in the present study are part of a larger set of neural regions active during piano performance (Bengtsson \& Ullén, 2006; Parsons, Sergent, Hodges, \& Fox, 2005). It would be of interest to examine to what extent these are specifically involved in creative behaviors in other domains.

\section{Acknowledgments}

We thank Lea Forsman, Hans Forssberg, Guy Madison, and Jeanne Nakamura for comments on the manuscript. This work was funded by the Swedish Research Council; the Freemasons in Stockholm Foundation for Children's Welfare, Sweden the Medici II symposia on positive psychology; the Templeton Foundation; and Linnea och Josef Carlssons Stiftelse, Sweden. The present address of S. L. B. is Wellcome Department of Imaging Neuroscience, Institute of Neurology, London, UK.

Reprint requests should be sent to Dr. Fredrik Ullén, Stockholm Brain Institute, Neuropediatric Research Unit Q2:07, Department of Women and Child Health, Karolinska Institutet, SE-171 76 Stockholm, Sweden, or via e-mail: Fredrik.Ullen@ki.se.

\section{REFERENCES}

Abdullaev, Y., \& Posner, M. I. (1997). Time course of activating brain areas in generating verbal associations. Psychological Science, 8, 56-59.

Andersen, R. A., Snyder, L. H., Bradley, D. C., \& Xing, J. (1997). Multimodal representation of space in the posterior parietal cortex and its use in planning movements. Annual Reviews of Neuroscience, 20, 303-330.

Ashburner, J., \& Friston, K. J. (1997). Multimodal image coregistration and partitioning-A unified framework. Neuroimage, 6, 209-217.

Baddeley, A. (1986). Working memory. Oxford: Clarendon Press.

Barbas, H., \& Pandya, D. N. (1987). Architecture and frontal cortical connections of the premotor cortex (area 6) in the rhesus monkey. Journal of Comparative Neurology, 256, 211-228.

Bates, J. F., \& Goldman-Rakic, P. S. (1993). Prefrontal connections of medial motor areas in the rhesus monkey. Journal of Comparative Neurology, 336, 211-228.

Bengtsson, S., Ehrsson, H. H., Forssberg, H., \& Ullén, F. (2004). Dissociating brain regions controlling the temporal and ordinal structure of learned movement sequences. European Journal of Neuroscience, 19, 2591-2602.

Bengtsson, S. L., Ehrsson, H. H., Forssberg, H., \& Ullén, F. (2005). Effector-independent voluntary timing: Behavioural and neuroimaging evidence. European Journal of Neuroscience, 22, 3255-3265.

Bengtsson, S. L., \& Ullén, F. (2006). Different neural correlates for melody and rhythm processing during 
piano performance from musical scores. Neuroimage, 30, 272-284.

Campbell, D. T. (1960). Blind variation and selective retention in creative thought as in other knowledge processes. Psychological Reviews, 67, 380-400.

Carlsson, I., Wendt, P. E., \& Risberg, J. (2000). On the neurobiology of creativity. Differences in frontal activity between high and low creative subjects. Neuropsychologia, 38, 873-885.

Csíkszentmihályi, M. (1997). Creativity: Flow and the psychology of discovery and invention. New York: Perennial.

Cummings, J. L. (1993). Frontal-subcortical circuits and human behavior. Archives of Neurology, 50, 873-880.

Deiber, M.-P., Passingham, R. E., Colebatch, J. G., Friston, K. J., Nixon, P. D., \& Frackowiak, R. S. J. (1991). Cortical areas and the selection of movement: A study with positron emission tomography. Experimental Brain Research, 84, 393-402.

Desmond, J. E., Gabrieli, J. D. E., \& Glover, G. H. (1998). Dissociation of frontal and cerebellar activity in a cognitive task: Evidence for a distinction between selection and search. Neuroimage, 7, 368-376.

Duvernoy, H. M. (2000). The buman brain: Surface, blood supply and three-dimensional sectional anatomy. Wien: Springer.

Eysenck, H. (1995). Genius. The natural history of creativity. Cambridge: Cambridge University Press.

Frith, C. D. (2000). The role of dorsolateral prefrontal cortex in the selection of action. In S. Monsell \& J. Driver (Eds.), Control of cognitive processes: Attention and performance (Vol. 18, pp. 429-565). Cambridge: MIT Press.

Frith, C. D., Friston, K. J., Liddle, P. F., \& Frackowiak, R. S. J. (1991). Willed action and the prefrontal cortex in man: A study with PET. Proceedings of the Royal Society of London, Series B, 244, 241-246.

Fuster, J. (2001). The prefrontal cortex-An update: Time is of the essence. Neuron, 30, 319-333.

Gaab, N., Gaser, C., Zaehle, T., Jancke, L., \& Schlaug, G. (2003). Functional anatomy of pitch memory-an fMRI study with sparse temporal sampling. Neuroimage, 19, 1417-1426.

Genovese, C. R., Lazar, N. A., \& Nichols, T. (2002). Thresholding of statistical maps in functional neuroimaging using the false discovery rate. Neuroimage, 15, 870-878.

Grafton, S. T., Fagg, A. H., \& Arbib, M. A. (1998). Dorsal premotor cortex and conditional movement selection: A PET functional mapping study. Journal of Neurophysiology, 79, 1092-1097.

Griffiths, T. D., Buchel, C., Frackowiak, R. S., \& Patterson, R. D. (1998). Analysis of temporal structure in sound by the human brain. Nature Neuroscience, 1, 422-427.

Hickok, G., Buchsbaum, B., Humphries, C., \& Muftuler, T. (2003). Auditory-motor interaction revealed by fMRI: Speech, music, and working memory in area Spt. Journal of Cognitive Neuroscience, 15, 673-682.

Hickok, G., \& Poeppel, D. (2000). Towards a functional neuroanatomy of speech perception. Trends in Cognitive Sciences, 4, 131-138.

Howard-Jones, P. A., Blakemore, S. J., Samuel, E. A., Summers, I. R., \& Claxton, G. (2005). Semantic divergence and creative story generation: An fMRI investigation. Cognitive Brain Research, 25, 240-250.

Jahanshahi, M., \& Dirnberger, G. (1999). The left dorsolateral prefrontal cortex and random generation of responses: Studies with transcranial magnetic stimulation. Neuropsychologia, 37, 181-190.

Jahanshahi, M., Dirnberger, G., Fuller, R., \& Frith, C. D. (2000). The role of the dorsolateral prefrontal cortex in random number generation: A study with positron emission tomography. Neuroimage, 12, 713-725.

Jahanshahi, M., \& Frith, C. D. (1998). Willed action and its impairments. Cognitive Neuropsychology, 15, 483-533.

Jahanshahi, M., Jenkins, I. H., Brown, R. G., Marsden, C. D., Brooks, D. J., \& Passingham, R. E. (1995). Self-initiated versus externally-triggered movements: Effects of stimulus predictability assessed with positron emission tomography. Journal of Psychophysiology, 9, 177-178.

Jahanshahi, M., Jenkins, I. H., Brown, R. G., Marsden, C. D., Passingham, R. E., \& Brooks, D. J. (1995). Self-initiated versus externally triggered movements: I. An investigation using measurement of regional cerebral blood flow with PET and movement-related potentials in normal and Parkinson's disease subjects. Brain, 118, 913-933.

Jahanshahi, M., Profice, P., Brown, R. G., Ridding, M. C., Dirnberger, G., \& Rothwell, J. C. (1998). The effects of transcranial magnetic stimulation over the dorsolateral prefrontal cortex on suppression of habitual counting during random number generation. Brain, 121, 1533-1544.

Jäncke, L., Loose, R., Lutz, K., Specht, K., \& Shah, N. J. (2000). Cortical activations during paced finger-tapping applying visual and auditory pacing stimuli. Cognitive Brain Research, 10, 51-66.

Johansen-Berg, H., Behrens, T. E., Robson, M. D., Drobnjak, I., Rushworth, M. F., Brady, J. M., et al. (2004). Changes in connectivity profiles define functionally distinct regions in human medial frontal cortex. Proceedings of the National Academy of Sciences, U.S.A., 101, 13335-13340.

Jueptner, M., Stephan, K. M., Frith, C. D., Brooks, D. J., Frackowiak, R. S., \& Passingham, R. E. (1997). Anatomy of motor learning: I. Frontal cortex and attention to action. Journal of Neurophysiology, 77, 1313-1324.

Knuth, D. E. (1981). Supernatural numbers. In D. A. Klarner (Ed.), The mathematical gardner (pp. 310-325). Belmont, CA: Wadsworth.

Larsson, J., Gulyás, B., \& Roland, P. E. (1996). Cortical representation of self-paced finger movement. NeuroReport, 7, 463-468.

Lau, H. C., Rogers, R. D., Ramnani, N., \& Passingham, R. E. (2004). Willed action and attention to the selection of action. Neuroimage, 21, 1407-1415.

Levenshtein, V. I. (1966). Binary codes capable of correcting deletions, insertions and reversals. Soviet Physics Doklady, 6, 707-710.

Lewis, P. A., Wing, A. M., Pope, P. A., Praamstra, P., \& Miall, R. C. (2004). Brain activity correlates differentially with increasing temporal complexity of rhythms during initialisation, synchronisation, and continuation phases of paced finger tapping. Neuropsychologia, 42, 1301-1312.

Lu, M. T., Preston, J. B., \& Strick, P. L. (1994). Interconnections between the prefrontal cortex and the premotor areas in the frontal lobe. Journal of Comparative Neurology, 341, 375-392.

Luria, A. R. (1966). Higher cortical functions in man. New York: Basic Books.

Lutz, K., Specht, K., Shah, N. J., \& Jäncke, L. (2000). Tapping movements according to regular and irregular visual timing signals investigated with fMRI. NeuroReport, 11, 1301-1306.

Macar, F., Lejeune, H., Bonnet, M., Ferrara, A., Pouthas, V., Vidal, F., et al. (2002). Activation of the supplementary motor area and of attentional networks during temporal processing. Experimental Brain Research, 142, 475-485.

Nathaniel-James, D. A., \& Frith, C. D. (2002). The role of the dorsolateral prefrontal cortex: Evidence from the effects 
of contextual constraint in a sentence completion task. Neuroimage, 16, 1094-1102.

Nichols, T., Brett, M., Andersson, J., Wager, T., \& Poline, J.-B. (2005). Valid conjunction inference with the minimum statistic. Neuroimage, 25, 653-660.

Ohbayashi, M., Ohki, K., \& Miyashita, Y. (2003). Conversion of working memory to motor sequence in the monkey premotor cortex. Science, 301, 233-236.

Oldfield, R. C. (1971). The assessment and analysis of handedness: The Edinburgh inventory. Neuropsychologia, 9, 97-113.

Palmer, K. (2001). Ornamentation according to C.P.E. Bach and J.J. Quantz. Bloomington, IN: Authorhouse.

Parsons, L. M., Sergent, J., Hodges, D. A., \& Fox, P. T. (2005). The brain basis of piano performance. Neuropsychologia, 43, 199-215.

Patterson, R. D., Uppenkamp, S., Johnsrude, I. S., \& Griffiths, T. D. (2002). The processing of temporal pitch and melody information in auditory cortex. Neuron, 36, 767-776.

Petersen, S. E., Fox, P. T., Posner, M. I., Nintus, M., \& Raichle, M. E. (1988). Positron emission tomographic studies of the cortical anatomy of single word processing. Nature, 331, 585-589.

Petrides, M. (1995). Impairments on nonspatial self-ordered and externally ordered working memory tasks after lesions of the mid-dorsal part of the lateral frontal cortex in the monkey. Journal of Neuroscience, 15, 359-375.

Picard, N., \& Strick, P. L. (2001). Imaging the premotor areas. Current Opinion in Neurobiology, 11, 663-672.

Playford, E. D., Jenkins, I. H., Passingham, R. E., Nutt, J., Frackowiak, R. S., \& Brooks, D. J. (1992). Impaired mesial frontal and putamen activation in Parkinson's disease: A positron emission tomography study. Annals of Neurology, 32, 151-161.

Pressing, J. (1988). Improvisation: Methods and models. In J. A. Sloboda (Ed.), Generative processes in music (pp. 129-178). New York: Oxford University Press.

Rowe, J. B., Stephan, K. E., Friston, K., Frackowiak, R. S., \& Passingham, R. E. (2005). The prefrontal cortex shows context-specific changes in effective connectivity to motor or visual cortex during the selection of action or colour. Cerebral Cortex, 15, 85-95.

Schmahmann, J. D., Doyon, J., Toga, A. W., Petrides, M., \& Evans, A. C. (2000). MRI atlas of the buman cerebellum. San Diego: Academic Press.

Schubotz, R. I., \& von Cramon, D. Y. (2001). Interval and ordinal properties of sequences are associated with distinct premotor areas. Cerebral Cortex, 11, 210-222.

Seger, C. A., Desmond, J. E., Glover, G. H., \& Gabrieli, J. D. (2000). Functional magnetic resonance imaging evidence for right-hemisphere involvement in processing unusual semantic relationships. Neuropsychology, 14, 361-369.

Simonton, D. K. (1999). Origins of genius. Darwinian perspectives on creativity. New York: Oxford University Press.

Sternberg, R. J. (1999). Handbook of creativity. Cambridge: Cambridge University Press.

Talairach, J., \& Tournoux, P. (1988). Co-planar stereotaxic atlas of the human brain. Stuttgart: Thieme.

Wise, S. P., Boussaoud, D., Johnson, P. B., \& Caminiti, R. (1997). Premotor and parietal cortex: Corticocortical connectivity and combinatorial computations. Annual Review of Neuroscience, 20, 25-42. 